\title{
A PRÁTICA DA ATENÇÃO FARMACÊUTICA NAS FARMÁCIAS COMUNITÁRIAS DE CURITIBA
}

\section{THE PHARMACEUTICAL CARE PRACTICE IN PHARMACIES OF CURITIBA CITY}

\author{
TORRES, Deborah Guiss ${ }^{1}$, MONTRUCCHIO, Deise Prehs ${ }^{2}$
}

\footnotetext{
1 Especialista em Atenção Farmacêutica - UFPR

2 Professora do Departamento de Farmácia - UFPR
}

RECEBIDO: JUL/07 ACEITE: OUT/07

\begin{abstract}
RESUMO
A Atenção Farmacêutica é uma prática profissional relativamente nova, que tem como foco principal o paciente e a meIhoria de sua qualidade de vida. Este estudo procurou analisar como se dá a prática da Atenção Farmacêutica na cidade de Curitiba, Paraná, levando às farmácias um caso simulado de potencial interação alimento-fármaco resultando em diminuição da dose do fármaco absorvida, causando efeito clínico indesejável por dose insuficiente de medicamento. Este pretenso paciente visitou quarenta farmácias da cidade de Curitiba no período de julho a setembro de 2006, solicitando orientações ao trabalhador que lhe atendia. Também foi objeto de estudo a presença ou não do farmacêutico na farmácia e sua visibilidade ao usuário como profissional da saúde: das quarenta farmácias visitadas, oito farmacêuticos apareceram prestando atendimento ao pretenso paciente. Houve uma baixa freqüência de atendimentos onde os trabalhadores destas farmácias deram respostas que do ponto de vista da Atenção Farmacêutica seriam desejáveis, ou que oferecessem Serviços Farmacêuticos, como Dispensação e Orientação Farmacêutica, Educação em Saúde ou Acompanhamento Farmacoterapêutico, e o foco principal foi na maioria, o medicamento. Também se verificou que não se procede a nenhum registro dos atendimentos nas farmácias curitibanas.
\end{abstract}

Palavras-chave: atenção farmacêutica; farmacêutico; orientação farmacêutica; dispensação.

\begin{abstract}
The Pharmaceutical Care is a relatively new professional practice which has as its main focus the patient and the improvement of his (her) quality of life. This study is the result of a research done in forty pharmacies in the city of Curitiba, Paraná, from July to September, 2006. A patient was sent looking for information and professional advice regarding possible food-drug interaction of her medication that resulted in low dosage drug therapy problem. This study was also used to determine how those pharmacies (in Curitiba) practice Pharmaceutical Care and to determine how the presence of the Pharmacist in the stores would impact or not this practice. Of the forty pharmacies in the study, only eight among them had a pharmacist present in the moment when the patient entered into the store and none of them wrote any registration.
\end{abstract}

Key words: pharmaceutical care; pharmacist; drug therapy problem.

\section{INTRODUÇÃO}

AAtenção Farmacêutica é uma prática profissional que representa um novo paradigma na Farmácia: a profissão farmacêutica deixando de ser unicamente voltada ao tecnicismo da droga e abrindo caminhos para centralizar o atendimento no paciente e em suas necessidades (MARIN, 2003:240; OLIVEIRA \& CHEMAKER, 2006:58). É uma prática profissional onde o farmacêutico toma para si a responsabilidade de gerenciar a farmacoterapia de seus pacientes, ofertando a eles serviços farmacêuticos, organizando a equipe prescritor-farmacêutico-paciente e colaborando na busca da melhoria da qualidade de vida deste paciente (PETRIS, 2006:04). 
Por volta de 1950 a Farmácia Hospitalar começou a estudar e desenvolver maneiras de distribuir o medicamento correto para o paciente correto no momento certo e a Farmácia Clínica tentava atingir este objetivo, também visando minimizar as intercorrências na distribuição de medicamentos industrializados nas farmácias comunitárias (MARIN, 2003:241). Na seqüência desse movimento, em 1990, Hepler e Strand apresentaram à comunidade científica um novo paradigma: pharmaceutical care, ou atenção farmacêutica, onde alertam farmacêuticos e suas instituições para parar de olhar para dentro de si mesmo e começar a redirecionar energias para um bem social maior, provendo pacientes com tratamento farmacológico responsável, com o propósito de alcançar resultados concretos que melhorem a qualidade de vida do paciente (HEPLER \& STRAND, 1990).

No Brasil a proposta do Consenso de Atenção Farmacêutica surgiu em 2002, fruto de diversas reuniões de grupos de profissionais, e colocou o conceito de Atenção Farmacêutica como um modelo de prática que acontece no contexto da Assistência Farmacêutica, envolvendo atitudes, valores éticos, comportamentos e habilidades envolvendo o farmacêutico com o usuário, mas sempre de forma integrada com a equipe de profissionais de saúde; como responsável na prevenção de doenças, promoção e recuperação da saúde (CONSENSO BRASILEIRO DE ATENÇÃO FARMACÊUTICA, 2002).

A Assistência Farmacêutica, por sua vez, é um ciclo de atividades relacionadas ao medicamento, englobando sua seleção, programação, aquisição, armazenamento, distribuição prescrição e dispensação, sempre preservando sua qualidade (MARIN, 2003). A dispensação, última etapa do ciclo, faz com que o farmacêutico seja o último profissional de saúde com o qual o paciente terá contato antes de iniciar sua farmacoterapia. Desta forma, cabe a este profissional fazer a orientação da melhor maneira de se utilizar este medicamento. A Organização Mundial da Saúde (OMS) vem discutindo exaustivamente o papel do farmacêutico no staff da saúde, reconhecendo que este é o profissional com o melhor perfil para condução de todas as ações destinadas a melhorar o acesso aos medicamentos essenciais e promover o seu uso racional (MARIN, 2003:124). Portanto, se faz necessário que o farmacêutico atuante em farmácias de dispensação siga o modelo de prática da Atenção Farmacêutica, aceitando a responsabilidade por otimizar toda a farmacoterapia de seu paciente, seja ela proveniente de uma prescrição, seja de automedicação ou de terapias alternativas, tudo em benefício da melhora na qualidade de vida do paciente (CIPOLLE, STRAND, MORLEY, 2004:02). A dispensação não pode ser a mera entrega do medicamento ao paciente, com discurso padronizado e despojado de qualquer adaptação às necessidades do complexo medicamento-paciente. Durante o ato da dispensação deve acontecer um processo de orientação farmacêutica, ato este "natural" do praticante da Atenção Farmacêutica que permite detectar um grande número de problemas relacionados com medicamentos (principalmente os riscos potenciais) (RIERA, 2004:1). Orientação Farmacêutica consiste em fornecer informações ao paciente com o objetivo de ajudá-lo a cumprir adequadamente um determinado regime medicamentoso (MARIN, 2003:255). Entretanto, existe um consenso universal que o autocuidado é o recurso primário de qualquer sistema de saúde: as pessoas procuram tratarem-se sozinhas, sem consultar nenhum profissional de saúde (WHO, 1998). Esta afirmativa da Organização Mundial de Saúde vem corroborar o quê se afirma neste trabalho, que o usuário busca frequentemente nas Farmácias de sua comunidade a automedicação necessária ao autocuidado. E quem deve estar preparado, disponível e visível a este usuário são os farmacêuticos, especialmente treinando e supervisionando sua equipe continuadamente. O usuário não vai aceitar somente o conselho de consultar um médico, sem saber o porquê. Até mesmo esta orientação deve ser fornecida adequadamente, com linguajar apropriado ao entendimento do usuário, por ser o conselho mais ético e correto, que não pode faltar no discurso do farmacêutico. Entretanto, o farmacêutico deve disponibilizar o máximo de informação possível que proporcione a automedicação responsável e estimule o usuário a cuidar bem de sua própria saúde, pois nem sempre o autocuidado envolve medicação (WHO, 1998).

Além disso, a legislação sanitária vigente no Brasil preconiza a presença do farmacêutico durante todo o horário de funcionamento da farmácia comunitária sob sua responsabilidade técnica, o que tem rigorosa fiscalização do Conselho Regional de Farmácia do Paraná (CRF - Pr) (ANVISA, 2001; CFF, 1997; CFF 2001). No entanto, a questão que se coloca é a seguinte: a presença do farmacêutico no estabelecimento é suficiente para assegurar uma dispensação bem orientada? Seguindo esta linha, esta pesquisa foi sobre a atuação dos farmacêuticos na cidade de Curitiba, Pr, sob o ponto de vista da Atenção Farmacêutica.

\section{MATERIAL E MÉTODOS}

Para analisar como se dá a Orientação Farmacêutica nas farmácias comunitárias de Curitiba, um instrumento de avaliação qualitativa com onze observações possíveis a um usuário foi elaborado especificamente para este fim, previamente estudado e testado em três farmácias piloto, sendo validado. Para tal, a autora apresentou-se como usuária de medicamento, explanando o seu caso e solicitando orientações. Imediatamente após o atendimento, o instrumento era preenchido e as observações anotadas. Para se determinar quais farmácias a serem 
avaliadas, foi obtida junto ao Conselho Regional de Farmácia do Paraná uma relação de todas as farmácias registradas em Curitiba. Esta lista foi então randomizada utilizando-se o programa "Math.random", um gerador de números aleatórios disponível em www.randomizer.org. Os dados coletados nas avaliações foram submetidos a uma análise percentual e distribuídos em gráficos.

Foram visitadas, entre junho e setembro de 2006, 40 farmácias em Curitiba, solicitando-se orientação em antibioticoterapia, resultante de automedicação. A usuária, com febre e dor de garganta, sexo feminino, por volta de 40 anos, encontrou em casa uma embalagem com três cápsulas de azitromicina, por algum motivo havia "sobrado" de um tratamento anterior de um parente com sintomas semelhantes aos seus e iniciou o tratamento, com uma cápsula ao dia, logo após o almoço, pois sabia que tomar medicamentos de estômago cheio evita "gastrite". Entretanto, após dois dias, achando que o efeito esperado não está acontecendo, procura a farmácia comunitária para orientações. "O que eu faço?", sem solicitar especificamente para falar com o farmacêutico.

\section{RESULTADOS}

Das 40 farmácias comunitárias visitadas, quase metade pertenciam a redes (19), quatro eram franquias, sete faziam parte de associações e dez eram independentes. Quanto ao profissional que prestou o primeiro atendimento, assim que a pretensa usuária entrou na farmácia, os dados informam que em trinta farmácias (75\%) os trabalhadores que prestaram o primeiro atendimento portavam crachás com seu nome, permitindo seu reconhecimento. Destes, três eram farmacêuticos, enquanto vinte e sete eram auxiliares (balconistas). Dez funcionários não estavam identificados. $O$ cruzamento destes dados, gera um resultado bastante interessante representado no gráfico 01: nas farmácias independentes a estrutura mais "enxuta" das empresas traz o farmacêutico mais para o balcão, mas é o tipo de farmácia em que seus trabalhadores estão mais pobremente identificados, o que não auxilia na popularização do farmacêutico.

\section{GRÁFICO 01: TIPO DE TRABALHADOR X TIPO DE FARMÁCIA}

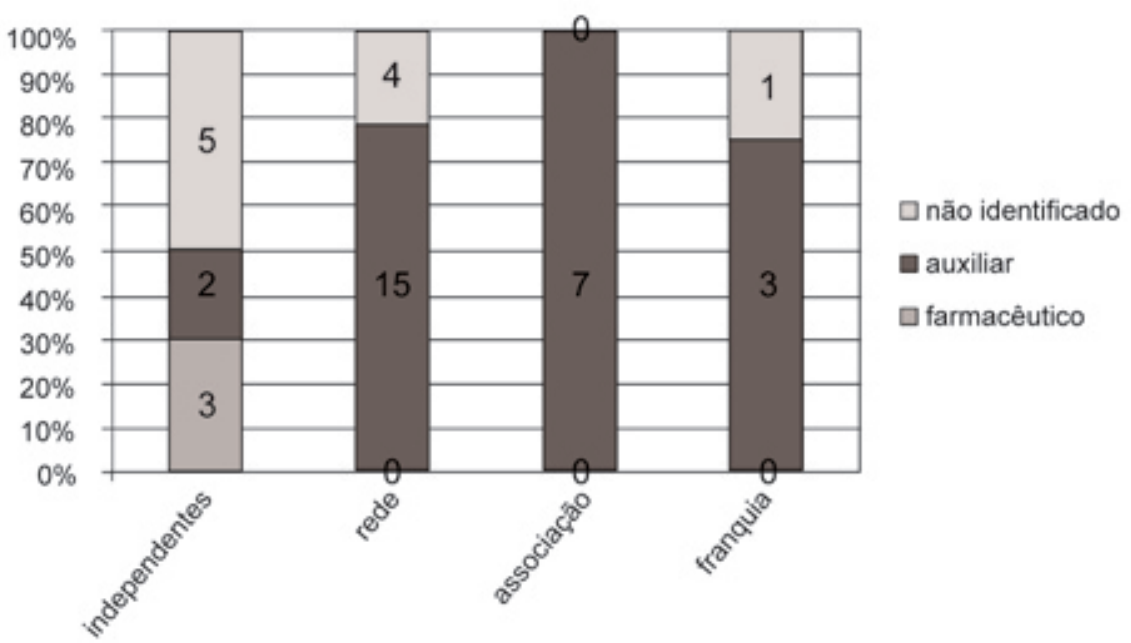

Observou-se que em 31 farmácias, (77\% do total), o farmacêutico não era visível na área de atendimento de pacientes. É difícil para a população reconhecer o trabalho de um profissional que não aparece. Junto a estes fatos, ainda o atendimento foi realizado pelo farmacêutico em três oportunidades e em duas, sob sua supervisão. A maioria dos atendimentos, porém, aconteceu sem a presença daquele profissional: $74 \%$ dos casos. Destes, apenas em um o farmacêutico foi acionado pelo auxiliar para dirimir dúvidas.

Em apenas doze atendimentos $(30 \%)$ houve investigação do problema relatado pela usuária. As perguntas mais freqüentes dos trabalhadores que prestaram o atendimento à pretensa paciente para investigar o problema aparecem no gráfico 02 , onde se nota claramente a preocupação se o medicamento é indicado àqueles sintomas, mas poucos atentaram a algum problema com a forma de administração: 


\section{GRÁFICO 02: PERGUNTAS MAIS FREQÜENTE NA INVESTIGAÇÃO DO PROBLEMA}

$\begin{array}{ll}\square \text { Quais seus sintomas } & \text { Quanto tempo tem os sintomas? } \\ \square \text { Jả foi ao médico? } & \square \text { Como toma? } \\ \square \text { Toma com o estômago cheio? } & \square \text { Qual horário? }\end{array}$

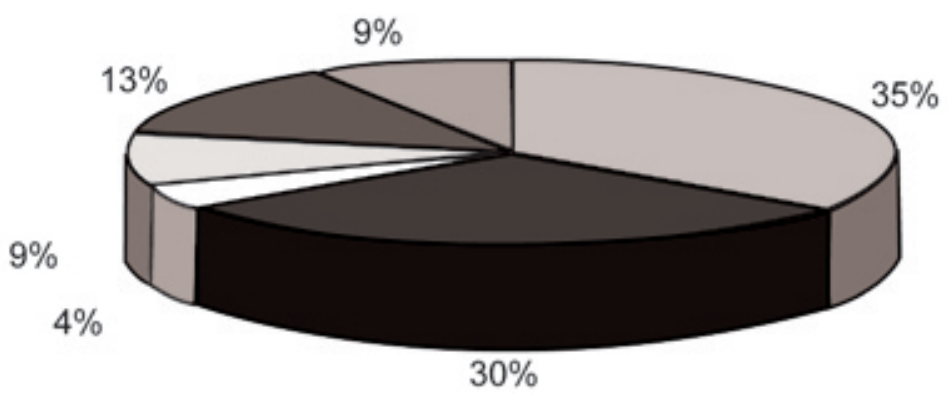

Quanto aos oito farmacêuticos envolvidos nos atendimentos, apenas um, o qual foi chamado pelo auxiliar, atentou ao problema relacionado com medicamento por dose insuficiente e orientou reiniciar o tratamento com cápsulas de azitromicina respeitando um intervalo do uma hora antes ou duas depois das refeições. Dois outros farmacêuticos encaminharam a usuária ao médico sem maiores orientações, três indicaram outro medicamento. Um dos farmacêuticos afastou-se para que o auxiliar orientasse a paciente sem supervisão alguma e outro farmacêutico orientou prolongar o tratamento por mais dois dias, sem, entretanto orientar modificação na maneira de administração. Dentre estes atendimentos, em apenas uma farmácia independente houve, por parte do farmacêutico, a oferta de outros serviços, como aferição de pressão arterial.

\section{DISCUSSÃO}

Nesta pesquisa ficou evidente que o farmacêutico não faz sua prática profissional popular e visível, apesar da fiscalização rigorosa do conselho de classe. Estão os colegas farmacêuticos aproveitando a oportunidade e arcando com a responsabilidade da Atenção Farmacêutica? Foram poucos os profissionais curitibanos vistos supervisionando suas áreas de venda no momento desta pesquisa, bem como poucos os que foram acionados por seus auxiliares, mostrando um grau de comprometimento pequeno das equipes das farmácias com a prática da Atenção Farmacêutica. Demonstrou-se que a prática não está centralizada no paciente, mas no medicamento e na venda: foram poucos os profissionais que souberam controlar a ansiedade de vendedor e tiveram tranqüilidade de ouvir a pretensa paciente em suas angústias e necessidades.

Em nosso país muito se discute sobre acessibilidade aos medicamentos, especialmente os essenciais. Mas para o consumidor, receber o medicamento e levá-lo para casa basta? Isto contribuirá para melhorar sua qualidade de vida? Os medicamentos, industrializados ou manipulados devem ser dispensado com Orientação Farmacêutica, sob a ótica da Atenção Farmacêutica, evitando Problemas Relacionados a Medicamentos (PRM's) e fazendo proveito dos investimentos do governo na aquisição de medicamentos para os postos de saúde, tornando os benefícios do fármaco acessíveis ao povo, como preconiza a Política Nacional de Medicamentos (BRASIL, 1998).

É necessário que os profissionais nas farmácias tenham ciência que estão prestando atendimento a seres humanos ávidos por informação e por saúde. Não há legislação que tome o lugar que de direito é do farmacêutico: a dispensação, pois envolve muito mais do que medicamentos industrializados e comissões. Envolve vidas e um enorme custo social de farmacoterapias mal sucedidas. Os currículos das escolas de Farmácia devem privilegiar a responsabilidade de assumir os riscos de cuidar de outro ser humano, de interagir com outros profissionais da equipe de saúde e especialmente a capacidade de amar a ponto de ouvir, entender e intervir. Esses currículos devem ser elaborados visando à inserção do farmacêutico na equipe de saúde, ou seja, que o formando saiba que ao sair da Escola irá trabalhar para o restabelecimento da saúde e para melhoria da qualidade de vida das pessoas e que os fármacos que ele tanto conhece são armas poderosas para obtenção destes objetivos, mas não as únicas. Como a Atenção Farmacêutica é uma prática bastante nova, não há muitos exemplos onde se 
espelhar, mas temos colegas que construíram práticas de atenção ao paciente de muito sucesso e que podem nos ensinar: enfermeiros, quiropráticos, veterinários, médicos, dentistas (CIPOLLE, STRAND, MORLEY, 2004:338).

E os profissionais que já estão atuando? Como melhorar o que se encontrou nesta pesquisa? Atualmente nenhum farmacêutico atua em farmácias em Curitiba sem que seu contrato de trabalho seja homologado pelo Conselho de Classe e pelo Sindicato de Farmacêuticos e a presença do Responsável Técnico é exaustivamente fiscalizada. Mas e a fiscalização sobre a sua atuação? Estes órgãos detêm o poder de fiscalização e seriam excelentes educadores dos farmacêuticos que já estão trabalhando, com cursos e eventos que os levassem a preocupar-se com o atendimento centrado no paciente e melhorassem a visão de si mesmos como profissionais de saúde. O CRF - Pr e o Sindicato (dos Farmacêuticos do Paraná) poderiam subsidiar a atualização dos profissionais, já que dominam a base da Legal desta prática, até que todos conhecessem este modelo de prática profissional. Conhecer a Atenção Farmacêutica é tão necessário hoje em oposição à baixa auto-estima trazida pela sensação de ter se tornado apenas um "profissional-instrumento", presente fisicamente somente para legalizar a prática deste comércio feroz e extremamente competitivo que leigos e indústria farmacêutica tornaram as farmácias comunitárias de Curitiba.

\section{REFERÊNCIAS}

ANVISA. Resolução n 357, de 20 de abril de 2001. Boas Práticas em Farmácias e Drogarias. Disponível em www.e-legis. bvs.br. Acesso em 11 de dezembro de 2005.

BRASIL. Consenso Brasileiro de Atenção Farmacêutica: Proposta. Ivama, Adriana et al. Organização Pan-Americana de Saúde. 2002.

BRASIL. Portaria $n^{\circ} 3916$ de 30/10/1998. Política Nacional de Medicamentos. Ministro de Estado de Saúde. D.O.U. nº 215-E seção 01 página 18 a 22, de 10/11/1998.

BRASIL. Lei n 5991 . Controle Sanitário de Drogas, Medicamentos, Insumos Farmacêuticos e correlatos. Congresso Nacional. D.O.U. de 19/12/1973.

CIPOLLE, ROBERT J., STRAND, LINDA M., MORLEY, PETER C..Pharmaceutical care Practice: the Clinician's Guide. 2 ed. U.S.A., The McGraw-Hill Companies, Inc. 2004.

CONSELHO FEDERAL DE FARMÁCIA. Resolução n 308, de 02/05/1997. Assistência Farmacêutica em Farmácias e Drogarias.

CONSELHO FEDERAL DE FARMÁCIA. Resolução n 357, 20/04/2001 Boas Práticas de Farmácia.. D.O.U. em 27/04/2001.

CONSELHO FEDERAL DE FARMÁCIA. Resolução n 417, de 29/09/2004. D.O.U. em 17/11/2004. Código de Ética da Profissão Farmacêutica.,

HEPLER, CHARLES, STRAND, LINDA. Opportunities and Responsabilities in the Pharmaceutical Care. American Journal Hosp. Pharmacists. 1990; 47: 533-543.

MARIN, NELLY et all. Assistência Farmacêutica para Gerentes Municipais. Rio de Janeiro; Organização Pan-Americana de Saúde/OMS, 2003.

OLIVEIRA, DJENANE R., SHOEMAKER, SARAH J. Achieving Patient centeredness in Pharmacy Practice. Journal of the American Pharmacist Association. Washington, D.C., 2006: 46 (1): 56-66

PARANÁ. Resolução n 54. NORMA Técnica para Abertura e Funcionamento de farmácias e Drogarias. Secretaria de Saúde do Estado do Paraná. Junho de 1996. D.O. estado n4378, de 14/06/1996.

RIERA, TERESA EYARALAR et al. Dispensación Activa. Aula de la Farmácia. Número 04, abril 2004. Disponível em www. auladelafarmacia.org. Acesso em 24 de julho de 2005.

VIVES, RAFAEL B..Sistemas Personalizados de Dispensación desde la Farmacia Comunitaria. Aula de la Farmácia. Número 20, novembro de 2005. www.auladelafarmacia.org. , 21 de dezembro de 2005.

URBIANIAK, GEOFFREY C. \& PLOUS, SCOTT. Disponível em www.randomizer.org. Acesso em 15/06/2006

WORLD HEALTH ORGANIZATION (1998). The role of the Pharmacist in Self-care and Self-medication. Hague, Neterlands, WHO/DAP/09, 13.

WORLD HEALTH ORGANIZATION. The Role of Pharmacists in the Health Care System, 1994. report of the WHO Meeting in Tokio, Japão, 31/08 a 03/09. WHO/PHARM/94, 569. 